\title{
THE SCATTERING OF RADIO WAVES IN THE SOLAR CORONA
}

\author{
A. HEWISH \\ Mullard Radio Astronomy Observatory, Cavendish Laboratory \\ Cambridge, England
}

The angular diameter of the Crab nebula is considerably increased when it is viewed through the solar corona. This effect is due to the scattering of the waves by coronal irregularities and its study gives some indication of the nature of the outermost corona. This paper is a summary of observations carried out each year at Cambridge for the period 1952 to 1958 inclusive.

\section{THE OBSERVATIONS}

Phase-switching interferometers were used at a variety of spacings and wavelengths to study the scattering over as wide a range of angular distances as possible. Observations at long wavelengths and with large interferometer spacings are necessary to study the small scattering in the outermost corona while shorter wavelengths must be used to measure the largest scattering at the distance of closest approach. Interferometers of only a few wavelengths spacing and a one-degree fan beam have been used to study changes of integrated intensity of the source. While most of the interferometers were aligned on an E.W axis some measurements have been carried out with the axes in other directions to investigate the scattering anisotropy.

The basic results are shown in Fig. 1, in which the apparent intensity of the source is plotted as a function of the date of observation. The general similarity of the curves from year to year is immediately apparent although greater reductions were observed toward sunspot maximum.

\section{INTERPRETATION OF THE OBSERVATIONS}

\section{(a) The magnitude of the scattering}

It has already been shown $[1,2]$ that the earlier results may be explained by a scattering mechanism which gives rise to scattered waves whose angular spectrum is of the form $\exp \left[-\left(\phi / \phi_{0}\right)^{2}\right]$ and the more recent results have fully confirmed this theory [3]. Values of $\phi_{0}$, the half-width of the angular spectrum, computed for the several results shown in Fig. 1 are plotted in Fig. 2.

\section{(b) Solar cycle variations}

In Fig. 3 the scattering at an angular distance of $8 R_{\odot}$ has been plotted as a function of the solar epoch. It is seen that the scattering falls to its least value in 1953-54, the period of sunspot minimum, and increases rapidly to a 


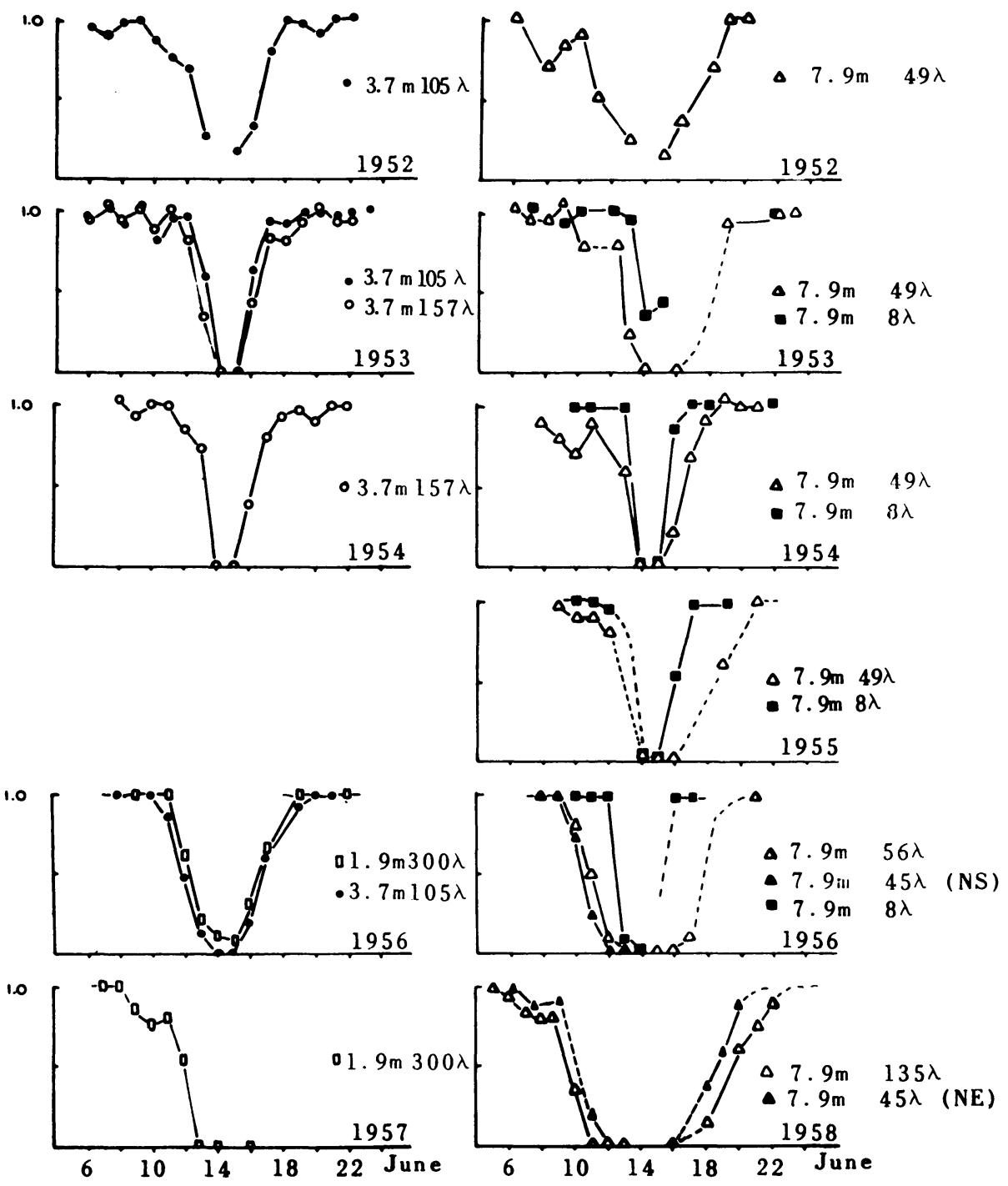

Fig. 1. The response of the Crab nebula observed with phase-switching interferometers of different wavelength and spacing.

maximum in 1957-58. At an angular distance of $16 R_{\odot}$, however, no pronounced variation is apparent and there appears to be no significant variation during the solar cycle. Since at a distance of $16 R_{\odot}$ we are concerned with scattering in the equatorial region of the corona and at $8 R_{\odot}$ with regions nearer the pole, these results strongly suggest that the distribution of the scattering irregularities follows the changing form of the visible corona, which tends to become more spherical toward sunspot maximum. 

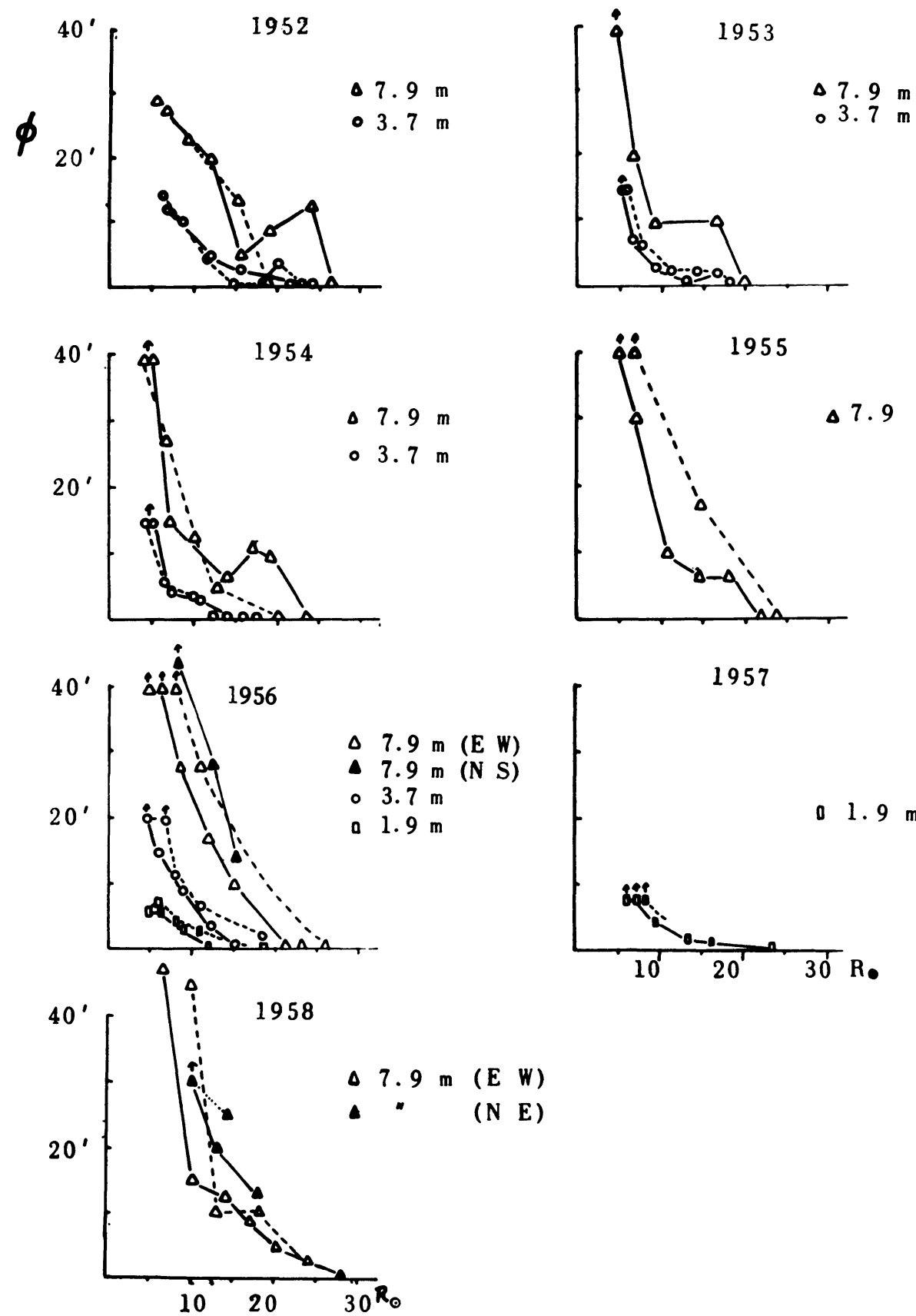

1957

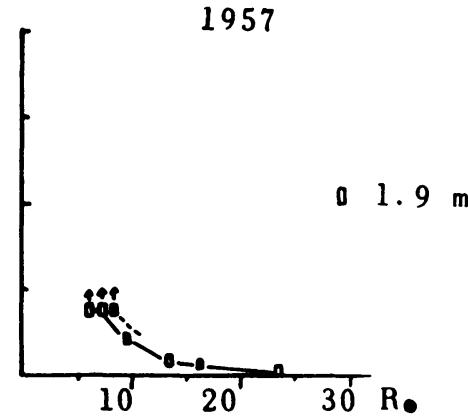

Fig. 2. Values of $\phi_{0}$, the half-width of the angular spectrum, at different wavelengths plotted against angular distance from the sun. 


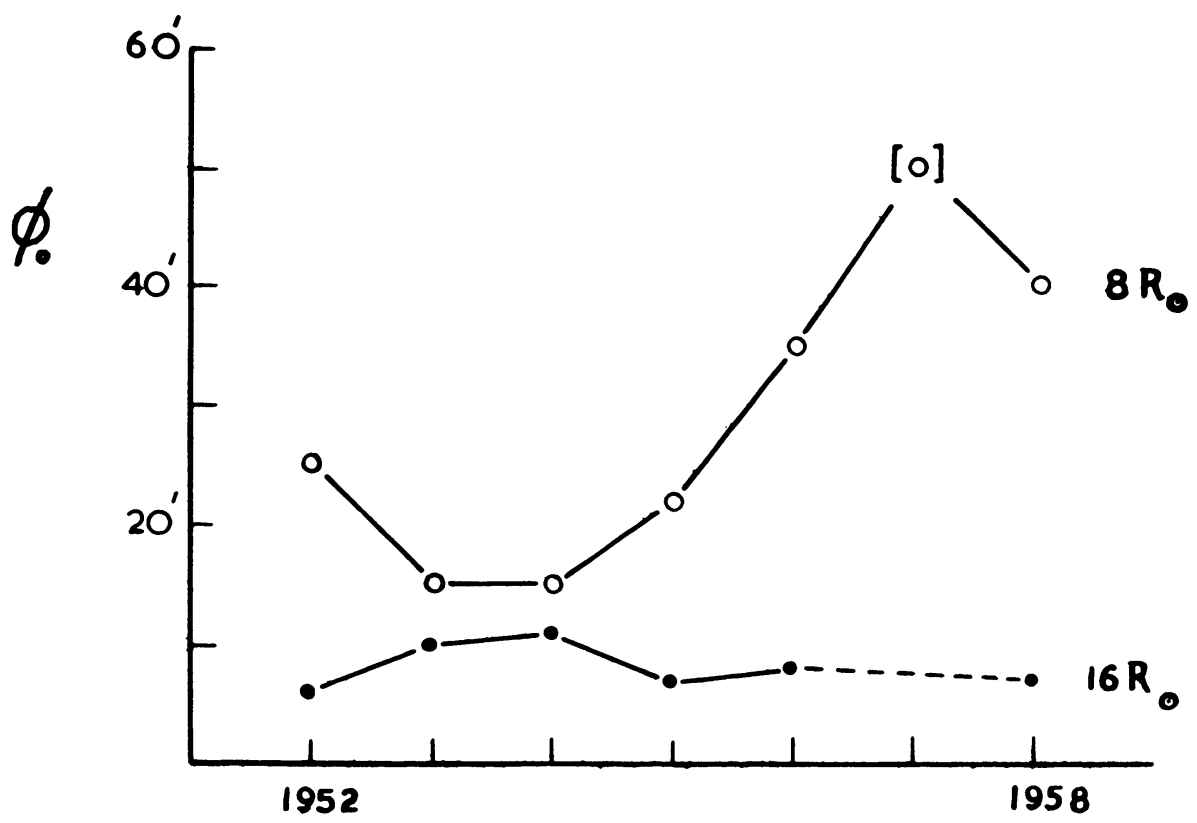

FIG. 3. The variation of scattering during the solar cycle.

(c) Modifications to the simple scattering theory at long wavelengths.

The simple scattering theory is not directly applicable to observations at wavelengths exceeding about $8 \mathrm{~m}$ for angular distances of less than about $8 R_{\odot}$ for two reasons: (1) The scattering is 80 great that the form of the scattered distribution is dependent upon the radial gradient of scattering; and (2) Effects due to refraction caused by the radial gradient of mean electron density become important.

In the absence of (2) computation indicates that the form of the scattered distribution at $7.9 \mathrm{~m}$ is similar to that shown in Fig. 4 . Since, however, scattered radiation cannot reach the earth when the ray deviation arising from (2) is greater than the scattering angle, it follows that some of the scattered distribution will be occulted leading to a reduction of integrated intensity. Observations at $7.9 \mathrm{~m}$ using interferometers of such short spacing that the scattering was always small compared with the lobe separation, have shown reductions of integrated intensity of at least 90 per cent at closest approach. Estimates based on

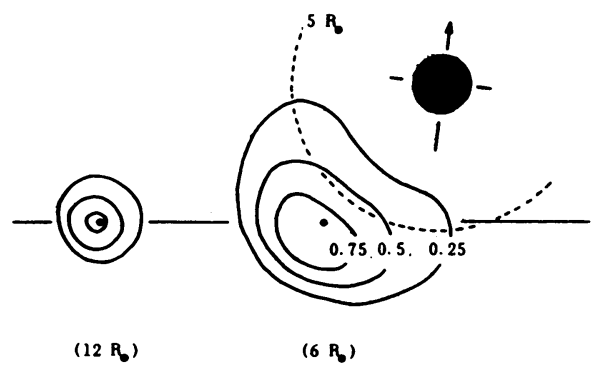

FIg. 4. The form of the scattered distribution at $7.9 \mathrm{~m}$ assuming isotropic scattering and a spherically symmetrical corona. 
Blackwell's optical data [4] indicate a refractive occultation radius of about $5 R_{\odot}$ which does not seem sufficient to account for the large observed reductions. It is likely that more detailed investigations will yield valuable data on the mean coronal density.

(d) Scattering by irregularities aligned in a magnetic field.

It was previously suggested that the coronal irregularities arose from diffusion of material along, but not across, the magnetic field as is believed to account for the visible polar plumes. Such a filamentary structure would lead to anisotropic scattering and experiments to detect this effect were carried out in 1956 using two interferometers of comparable spacing with N-S and E-W axes, and in 1958 with three interferometers aligned on E-W, N-E, and $\mathrm{N} \cdot \mathrm{W}$ axes. The scattering was found to be considerably greater in directions parallel to the solar axis than perpendicular to it. The results, interpreted as elliptical scattering contours, are shown in Fig. $5(b)$.

To compare these results with what might be expected theoretically in a given case, computations were carried out on the assumption that the sun's
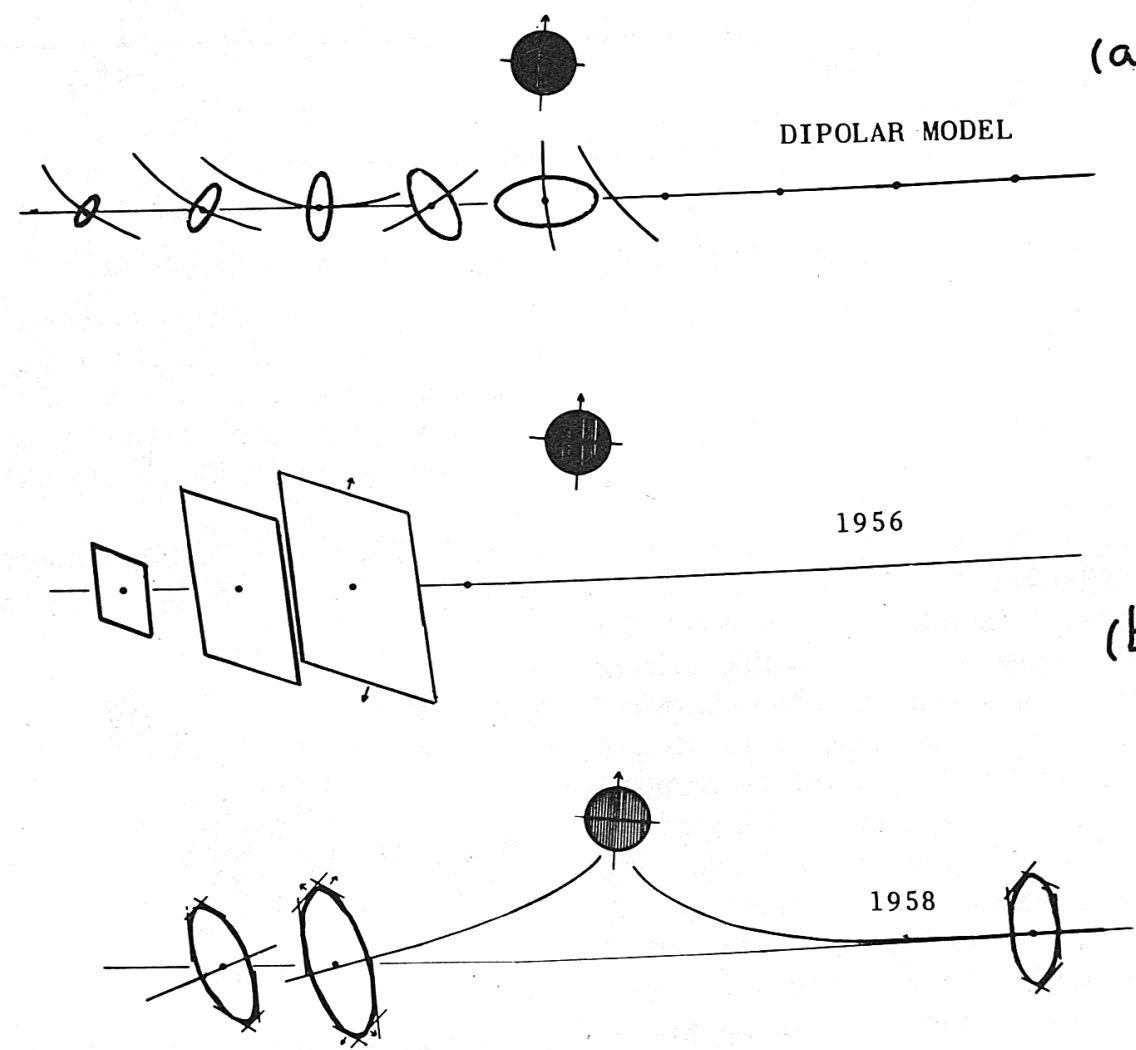

FIG. 5. (a) The theoretical scattering anisotropy computed for a dipolar model of magnetic field. (b) The observed scattering anisotropy. 
general magnetic field is dipolar. It was further assumed that the electron density corresponded to the observations of Blackwell [4]. The scattering computed for this model is shown in Fig. 5 (a). Comparison with the experimental results shows that while the scattering anisotropy is of the right order of magnitude the inclination of the ellipses is wrong. The observations indicate a general magnetic field directed approximately parallel to the equatorial plane at distances exceeding $10 R_{\odot}$.

\section{REFERENCES}

[1] Hewish, A. Proc. Roy. Soc. A 228, 238, 1955.

[2] Vitkevich, V. V. Dokl. Acad. Nauk S.S.S.R. 101, 429, 1955.

[3] Hewish, A. The Observatory, 77, 151, 1957.

[4] Blackwell, D. E. M.N.R.A.S. 115, 629, 1955.

\section{Discussion}

Gold: The idea of a greatly stretched-out magnetic field in or near the sun's equatorial plane has been proposed for a number of compelling reasons, and I am glad to have a further indication of agreement with it. Could you explain why the lens effect cuts out the signal rather than provides merely different paths?

Hewish: The radial gradient of electron density in the corona makes it act like a large diverging lens. For any point within the caustic surface the Crab nebula would be completely occulted in the absence of scattering. The caustic surface is blurred by the large scattering, and occultation can only occur at smaller angular distances when the ray deviation is greater than the scattering angle.

Denisse: It is worth mentioning in this connection that Boischot finds an increase instead of a decrease in the total intensity received from the occulted Crab nebula.

van de Hulst: What is the basis of the theoretical prediction of the ellipticity of the scattering ellipse?

Hewish: We assumed that in each volume-element the scattering is perpendicular to the magnetic field; the rest is a nasty integration over the line of sight.

van de Hulst: Would you not, at the point where you cross the sun's axis, get a very elongated ellipse?

Hewish: Any individual filament scatters strongly in directions perpendicular to its axis and particular filaments are tangential to the line of sight; this gives an elliptical distribution of scattered radiation.

van de Hulst: Has anybody obtained results for wavelengths longer than $8 \mathrm{~m}$ ?

Stanley: We have also observed an occultation at $26.4 \mathrm{Mc} / \mathrm{s}$ this June. The sun was extremely quiet throughout the occultation and for several weeks before and afterward. The intensity of the Crab nebula was reduced to 30 per cent of its normal value throughout the eclipse and the sun's diameter was approximately 8 degrees. 